\title{
CLINICAL DEVELOPMENT PLAN OF NEW MOLECULES FOR TARGETING CHRONIC NEUROPATHIC PAIN. THE PLACEBO ARM IS VITAL SINCE EARLY TRANSLATIONAL RESEARCH TO COMPLETION OF PHASE 3
}

D Merante ${ }^{1}$, S Patel ${ }^{2}$.

${ }^{1}$ Pharmaceutical Physician, Global Clinical Development, Amersham, United Kingdom;

2Pharmaceutical Physician, Global Clinical Development, London, United Kingdom

\section{Background and Aims:}

The inclusion of placebo in clinical studies allows to detect if an investigational medication is superior to placebo in terms of NRS/VAS pain scoring (the most conventional study primary endpoint). Randomized, double blind, placebo controlled studies represent the gold standard in Chronic Neuropathic Pain (CNP) clinical research. Traditionally placebo controlled randomized studies are globally recommended by current regulatory guidelines. Aims of this research was to analyse the clinical relevance of placebo arm in more innovative study designs in CNP. The primary scope of this research was to explore such study designs but reducing/mitigating the burden of a large placebo response, which is often the main reason of study or program failures in CNP trials.

\section{Methods:}

An extensive research of studies in phase 1-2-3 of clinical development in PUBMED and MEDLINE was carried out from to 2000 up to date to look, based on the final outcome, at more innovative study designs in CNP.
Results:

Conventionally, Single Ascending and Multiple Ascending, as well as a Food Effect and a Multi-Ethnic studies (parallel or cross-over) require a placebo comparative arm. In these studies a new molecule is pharmacologically investigated at different doses. Safety and tolerability is carefully assessed since early clinical development. In phase 1 a placebo arm and an active comparator arm should be considered in pain-induced model studies (i.e. capsaicin studies) in healthy volunteers. In addition to placebo, in phase 2 and 3 an active comparator is also utilized in study designs. This is relevant for any newly investigated molecule to assess the analgesic and tolerability profile vs. placebo and the active comparator. In addition, a cross-over design should be considered in CNP paediatric studies.

\section{Conclusions:}

We found that randomized adaptive and withdrawal placebo controlled trials are good examples of innovative study designs in CNP (refer to mirogabalin phase 2 and tapentandol phase 3 in diabetic painful neuropathy and the sodium channel blocker CNV1014802 phase 2 in trigeminal neuralgia, a very severe form of facial pain).

\section{Examples of innovative study designs in CNP with the placebo arm as primary comparator}
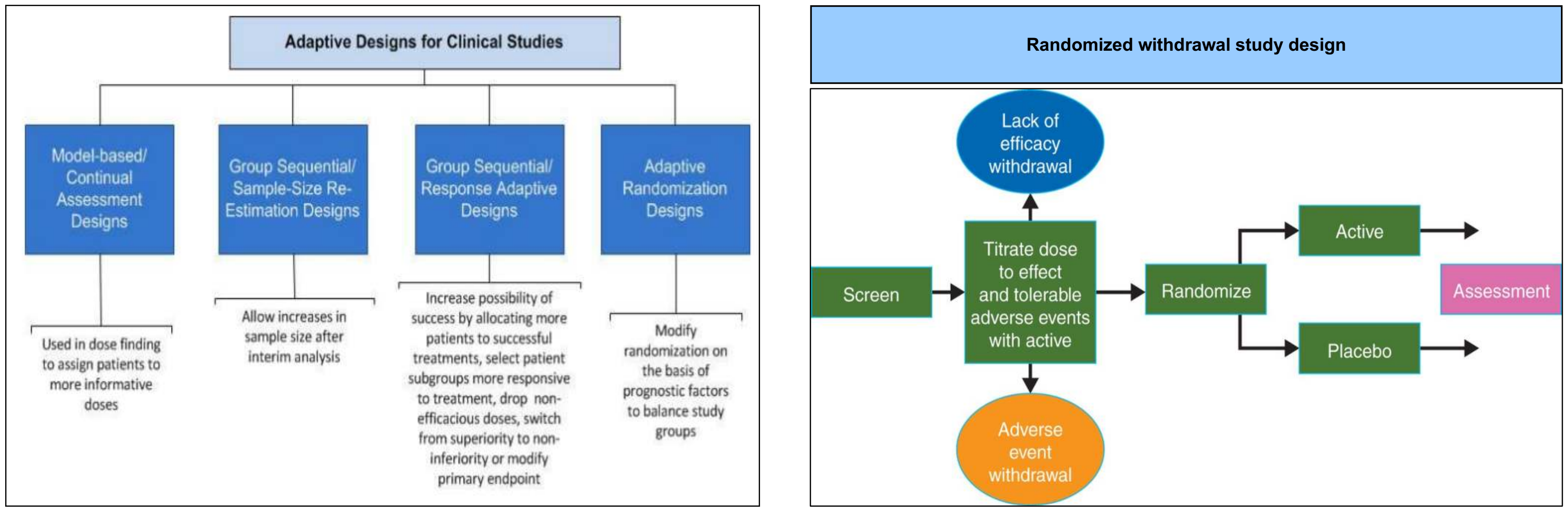